
Applied and
Computational
Mathematics
Division

NISTIR 4956

Computing and Applied Mathematics Laboratory

\title{
Thermodynamically-Consistent Phase-Field Models for Solidification
}

\author{
S-L. Wang, R. F. Sekerka, A. A. Wheeler, \\ B. T. Murray, S. R. Coriell, R. J. Braun, \\ and G. B. McFadden
}

November 1992

Technology Administration

U.S. DEPARTMENT OF COMMERCE

National Institute of Standards and Technology

Gaithersburg, MD 20899 



\section{Thermodynamically-Consistent Phase-Field Models for Solidification}

S-L. Wang
R. F. Sekerka
A. A. Wheeler
B. T. Murray
S. R. Coriell
R. J. Braun
G. B. McFadden

U.S. DEPARTMENT OF COMMERCE Technology Administration National Institute of Standards and Technology

Computing and Applied Mathematics Laboratory

Gaithersburg, MD 20899

November 1992

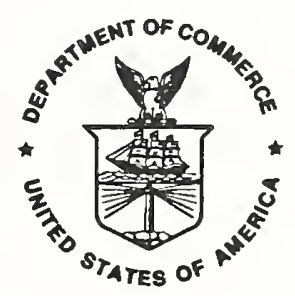

U.S. DEPARTMENT OF COMMERCE Barbara Hackman Franklin, Secretary

TECHNOLOGY ADMINISTRATION Robert M. White, Under Secretary for Technology

NATIONAL INSTITUTE OF STANDARDS AND TECHNOLOGY

John $W$. Lyons, Director 



\title{
Thermodynamically-Consistent Phase-Field Models for Solidification
}

\author{
S-L. Wang and R. F. Sekerka* \\ Department of Physics \\ Carnegie Mellon University \\ Pittsburgh, PA 15213 USA
}

\author{
A. A. Wheeler \\ School of Mathematics \\ University of Bristol \\ Bristol BS8 1TW, U.K.
}
B. T. Murray, S. R. Coriell, R. J. Braun, and G. B. McFadden National Institute of Standards and Technology ${ }^{\dagger}$ Gaithersburg, MD 20899 USA

\begin{abstract}
In an effort to unify the various phase-field models that have been used to study solidification, we have developed a class of phase-field models for crystallization of a pure substance from its melt. These models are based on an entropy functional, as in the treatment of Penrose and Fife, and are therefore thermodynamically consistent inasmuch as they guarantee spatially local positive entropy production. General conditions are developed to ensure that the phase field takes on constant values in the bulk phases. Specific forms of a phase-field function are chosen to produce two models that bear strong resemblances to the models proposed by Langer and Kobayashi. Our models contain additional nonlinear functions of the phase field that are necessary to guarantee thermodynamic consistency.
\end{abstract}

PACS numbers: $64.70 . \mathrm{Dv}, 68.10 . \mathrm{Gw}$, 81.30.Bx, 82.65.Dp

Keywords: non-equilibrium thermodynamics, phase field, solidification, free boundary, entropy functional, Model C

${ }^{\dagger}$ Technology Administration, U.S. Department of Commerce, Washington D.C. 


\section{Introduction}

The classical approach to the modeling of first order phase transformations involves tracking of the free boundary that separates the growing phase from the parent phase. This requires the solution of a formidable free boundary problem. For example, for crystallization of a solid (crystal) from a pure liquid (melt), one must solve the equations for the temperature fields in both solid and liquid subject to boundary conditions for the temperature and its derivatives on the moving solid-liquid interface, the free boundary.

The phase-field model provides an alternative approach, according to which a new variable, the phase field $\phi$, is introduced to keep track of the phase, taking on constant values indicative of each of the bulk phases and making a transition between these values over a thin transition layer that plays the role of the classically sharp interface. The phase field $\phi$ is governed by a partial differential equation that guarantees (in the asymptotic limit of a suitably thin transition layer) that the appropriate boundary conditions at the crystal-melt interface are satisfied. Moreover, the transport equations are modified, by addition of terms that depend on the phase field, to apply in all of space and to embody the conservation conditions at the interface. One then proceeds to solve the coupled equations for the phase field and transport-related fields (e.g., temperature and/or composition) without the necessity of explicit tracking of the free boundary.

Langer [1,2] adapted Model C of Halpern, Hohenberg, and Ma [3] to produce a phasefield model that accounts for the Gibbs-Thomson equation at the solid-liquid interface for solidification problems. Moreover, a similar phase-field model was introduced by Collins and Levine [4]. Analytical properties of the phase-field equations resulting from Langer's model have been analyzed extensively by Caginalp et al. [5-13], Gurtin [14] and Fife and Gill $[15,16]$. In particular, Caginalp [11] has shown that certain distinguished limits of the phase-field equations lead to various free boundary problems, including the classical models of solidification.

Numerical methods for computation using a phase-field model were introduced by Fix [17] and early numerical computations in one dimension were performed by Lin [18]. 
Subsequently, there have been a number of computations in one dimension, including those by Schofield and Oxtoby [19] for planar geometries and by Caginalp and Socolovsky [12] for planar and spherically symmetric geometries. Success with numerical computations for more complex shapes has been limited because of the considerable computational power needed to resolve the diverse length and time scales intrinsic to the phase-field model. Recently, Kobayashi [20] introduced a phase-field model that is particularly suitable for numerical computations of the dendritic solidification of a pure material from a supercooled melt, in two $[21,22]$ and three [23] dimensions. Using the phase-field model described here, numerical computations in two dimensions have been carried out for the dendritic growth of nickel by Wheeler et al. [24].

A difficulty with phase-field models, as pointed out by Penrose and Fife [25], lies in their derivation. In particular, the governing equation for the phase field is usually derived from a free energy functional that is applicable only to an isothermal situation; then the classical equation for the temperature field is altered in an ad hoc manner to account for the liberation of latent heat by addition of a term proportional to the time derivative of the phase field. Penrose and Fife, however, provided a framework from which the equations for the phase field and the temperature can be derived in a thermodynamically consistent manner from a single entropy functional.

In the present paper, we employ the method of Penrose and Fife to derive a class of phase-field models for the crystallization of a pure material from its melt that have the following properties:

1. They are based on an entropy functional and guarantee local positive entropy production,

2. The functions and parameters in the model can be chosen to agree with empirical data, such as specific heats and surface tension, asymptotically in the classical limit,

3. They satisfy criteria to insure that the phase field will take on fixed values, which we take to be 0 for the solid and 1 for the liquid, for the bulk phases, 
4. They allow for specific choices of the phase-field function that lead to models, which we call Model I and Model II, that bear a strong resemblance to the models of Langer and Kobayashi, respectively, but that display specific nonlinearities necessary to satisfy properties (1-3). Thus, we provide a common basis for their models.

\section{Thermodynamic Formulation}

For purposes of deriving the partial differential equations for the phase-field model, we consider a closed system of volume $V$ in which a pure material undergoes a first-order phase transition between solid and liquid. We introduce an order parameter $\phi(\mathbf{x}, t)$, called the phase field, that indicates the phase of the material at $(x, t)$. We assume that $\phi$ equals zero in the solid phase and unity in the liquid phase. Thin regions, in which $\phi$ takes values between zero and unity, will be shown to correspond to crystal-melt interfaces. For simplicity, we assume uniform density throughout the system and that there is no convection in the liquid; this ensures mechanical equilibrium.

We assume that the internal energy of any subvolume $\mathcal{V}$ of $V$ is represented by the integral

$$
\mathcal{E}=\int_{\mathcal{V}} e d v
$$

According to the first law of thermodynamics, the time rate of change of $\mathcal{E}$ obeys

$$
\dot{\mathcal{E}}+\int_{\mathcal{A}} \mathbf{q} \cdot \mathbf{n} d a=0
$$

where $\mathcal{A}$ is the surface of $\mathcal{V}$ with outward normal $\mathbf{n}$, and $\mathbf{q}$ is flux of internal energy. Converting the surface integral in Eq. (2) to a volume integral by Gauss' theorem and equating the integrands, which is permissible since $\mathcal{V}$ is arbitrary, leads to the familiar equation

$$
\dot{e}+\nabla \cdot \mathbf{q}=0 .
$$

The entropy of any subvolume $\mathcal{V}$ of $V$ is assumed to be represented by the functional

$$
\mathcal{S}=\int_{\mathcal{V}}\left[s-\frac{\epsilon^{2}}{2}(\nabla \phi)^{2}\right] d v
$$


where $s(e, \phi)$ is an entropy density (an extension of classical thermodynamics) that would apply to a uniform system of internal energy density $e$ and phase field $\phi$, and $\epsilon$ is a constant. The second term in the integrand is a gradient entropy term analogous to the Landau-Ginzburg or Cahn-Hilliard gradient energy term in the free energy, cf. Eq. (37). Differentiation of Eq. (4) with respect to time gives

$$
\begin{gathered}
\dot{\mathcal{S}}=\int_{\mathcal{V}}\left\{\left(\frac{\partial s}{\partial e}\right)_{\phi} \dot{e}+\left[\left(\frac{\partial s}{\partial \phi}\right)_{e}+\epsilon^{2} \nabla^{2} \phi\right] \dot{\phi}-\epsilon^{2} \nabla \cdot(\dot{\phi} \nabla \phi)\right\} d v \\
=\int_{\mathcal{V}}\left\{\mathbf{q} \cdot \nabla\left(\frac{\partial s}{\partial e}\right)_{\phi}+\left[\left(\frac{\partial s}{\partial \phi}\right)_{e}+\epsilon^{2} \nabla^{2} \phi\right] \dot{\phi}\right\} d v \\
-\int_{\mathcal{A}}\left[\left(\frac{\partial s}{\partial e}\right)_{\phi} \mathbf{q}+\epsilon^{2} \dot{\phi} \nabla \phi\right] \cdot \mathbf{n} d a,
\end{gathered}
$$

where we have substituted for $\dot{e}$ from Eq. (3) and used Gauss' theorem to integrate by parts.

The second law of thermodynamics requires the entropy production to be positive in any subvolume $\mathcal{V}$ of $V$. This entropy production can be calculated by subtracting from $\dot{\mathcal{S}}$ the entropy flux through $\mathcal{A}$, which leads to the relation

$$
\dot{\mathcal{S}}+\int_{\mathcal{A}}\left\{\frac{\mathrm{q}}{T} \cdot \mathbf{n}+\epsilon^{2} \dot{\phi} \nabla \phi \cdot \mathbf{n}\right\} d a \geq 0
$$

where $T$ is the absolute temperature. In Eq. (6), $\mathrm{q} / T$ is the familiar entropy flux due to heat flow; the quantity $\epsilon^{2} \dot{\phi} \nabla \phi$ is an entropy flux related to changes in the phase field at the boundary of the subvolume $\mathcal{V}$. The volumetric entropy production and the entropy flux associated with $\phi$ will be related to their counterparts for a sharp-interface model in the discussion section.

Substitution of Eq. (5b) into Eq. (6) then leads to

$$
\int_{\mathcal{V}}\left\{\mathbf{q} \cdot \nabla\left(\frac{1}{T}\right)+\left[\left(\frac{\partial s}{\partial \phi}\right)_{e}+\epsilon^{2} \nabla^{2} \phi\right] \dot{\phi}\right\} d v \geq 0
$$

where we have used

$$
d e=T d s+\left(\frac{\partial e}{\partial \phi}\right)_{s} d \phi
$$


to identify $(\partial s / \partial e)_{\phi}=(1 / T)$. We can therefore guarantee local positive entropy production by choosing

$$
\begin{aligned}
\mathbf{q} & =M_{T} \nabla\left(\frac{1}{T}\right), \\
\tau \dot{\phi} & =\left(\frac{\partial s}{\partial \phi}\right)_{e}+\epsilon^{2} \nabla^{2} \phi
\end{aligned}
$$

where $M_{T}$ and $\tau$ are positive. This choice allows the integrand in Eq. (7) to be reduced to a sum of squares: $|\mathbf{q}|^{2} / M_{T}+\tau(\dot{\phi})^{2}$. The governing equations therefore become

$$
\dot{e}=-\nabla \cdot\left[M_{T} \nabla\left(\frac{1}{T}\right)\right]
$$

for the internal energy density, and

$$
\tau \dot{\phi}=-\frac{1}{T}\left(\frac{\partial e}{\partial \phi}\right)_{s}+\epsilon^{2} \nabla^{2} \phi
$$

for the phase field.

In order to identify the derivative in Eq. (12), it is convenient to work with the Helmholtz free energy density

$$
f=e-T s,
$$

whose differential, when combined with Eq. (8), yields

$$
\left(\frac{\partial e}{\partial \phi}\right)_{s}=\left(\frac{\partial f}{\partial \phi}\right)_{T}
$$

and

$$
\left(\frac{\partial[f / T]}{\partial T}\right)_{\phi}=-\frac{e}{T^{2}}
$$

The latter can be integrated at constant $\phi$ to give

$$
f(T, \phi)=T\left[-\int_{T_{M}}^{T} \frac{e(\zeta, \phi)}{\zeta^{2}} d \zeta+G(\phi)\right],
$$

where $G(\phi)$ is an undetermined function of $\phi$ and $T_{M}$ is the melting point. To proceed further, we assume that the internal energy density has the form

$$
e=e_{S}(T)+p(\phi) L(T)=e_{L}(T)+[p(\phi)-1] L(T)
$$


where $e_{S}(T)$ and $e_{L}(T)$ are the classical internal energy densities of the solid and liquid phases, respectively, and $L(T)=e_{L}(T)-e_{S}(T)$ is their difference. Here $p(\phi)$ satisfies $p(0)=0$ and $p(1)=1$. We note that $L_{0} \equiv L\left(T_{M}\right)$ is the latent heat of fusion; whereas the variation of $L(T)>0$ with temperature allows the specific heats of solid and liquid to depend differently on temperature, as illustrated in Figure 1. From Eq. (16), the free energy density corresponding to this internal energy density is

$$
f(T, \phi)=T\left[-\int_{T_{M}}^{T} \frac{e_{L}(\zeta)}{\zeta^{2}} d \zeta-[p(\phi)-1] Q(T)+G(\phi)\right]
$$

where

$$
Q(T)=\int_{T_{M}}^{T} \frac{L(\zeta)}{\zeta^{2}} d \zeta
$$

We note that $Q(T)$ is monotonic increasing with respect to its argument, $T$, and $Q\left(T_{M}\right)=$ 0. From Eq. (14) and Eq. (18) we have

$$
\left(\frac{\partial e}{\partial \phi}\right)_{s}=-T Q(T) p^{\prime}(\phi)+T G^{\prime}(\phi)
$$

Thus, the governing equations, Eq. (11) and Eq. (12), become

$$
\dot{e}_{L}(T)+\dot{p}(\phi) L(T)+[p(\phi)-1] \dot{L}(T)=-\nabla \cdot\left[M_{T} \nabla\left(\frac{1}{T}\right)\right]
$$

and

$$
\tau \dot{\phi}=Q(T) p^{\prime}(\phi)-G^{\prime}(\phi)+\epsilon^{2} \nabla^{2} \phi
$$

We have assumed that $\phi=0$ and $\phi=1$ correspond to bulk solid and liquid states for all values of the temperature. This consideration requires $f$ to have, for all values of $T$, local minima with respect to $\phi$ at $\phi=0$ and $\phi=1$. We also require $f$ to be continuous with respect to the temperature at the melting point; therefore

$$
\begin{aligned}
f\left(T_{M}, 0\right) & =f\left(T_{M}, 1\right) \\
\left.\frac{\partial f}{\partial \phi}\right|_{\phi=0,1} & =0 \\
\left.\frac{\partial^{2} f}{\partial \phi^{2}}\right|_{\phi=0,1} & >0
\end{aligned}
$$


These conditions require that

$$
\begin{aligned}
G(0) & =G(1) \\
{\left.\left[G^{\prime}(\phi)-p^{\prime}(\phi) Q(T)\right]\right|_{\phi=0,1} } & =0 \\
{\left.\left[G^{\prime \prime}(\phi)-p^{\prime \prime}(\phi) Q(T)\right]\right|_{\phi=0,1} } & >0 .
\end{aligned}
$$

Accordingly we choose $G(\phi)$ to be a symmetric 'double well' potential with minima at zero and unity of the form

$$
G(\phi)=\frac{1}{4 a} g(\phi)
$$

where

$$
g(\phi)=\phi^{2}(1-\phi)^{2},
$$

and $a$ is a positive constant; this choice implies the convention $f\left(T_{M}, 0\right)=f\left(T_{M}, 1\right)=0$. In the next section we explore two possible choices for $p(\phi)$ which lead to phase-field models that may be compared to those of Langer and Kobayashi.

We continue with the general development of the phase-field equations by further specifying the thermodynamic description of the system. For a pure material, one is mostly interested in studying morphological instabilities and subsequent dendritic growth that can occur in solidification from an undercooled melt, where heat conduction takes place mostly in the liquid. Therefore, in order to obtain a tractable problem, we assume the energy density in the liquid to be a linear function of temperature of the form

$$
e_{L}(T)=e_{L}\left(T_{M}\right)+c\left(T-T_{M}\right)
$$

where $c$ is a constant specific heat, which will lead to the classical heat equation in the bulk liquid. Eq. (21) and Eq. (22) therefore become

$$
\left\{c+(p(\phi)-1) L^{\prime}(T)\right\} \frac{\partial T}{\partial t}+L(T) p^{\prime}(\phi) \frac{\partial \phi}{\partial t}=k \nabla^{2} T,
$$

and

$$
\tau \frac{\partial \phi}{\partial t}=Q(T) p^{\prime}(\phi)-\frac{1}{4 a} g^{\prime}(\phi)+\epsilon^{2} \nabla^{2} \phi
$$

where we have set $M_{T}=k T^{2}$. The thermal conductivity $k$ is assumed to be constant. 
At this point in the development of the equations, the parameters $a$ and $\epsilon$ can be related to the interface thickness and surface free energy by examining a one-dimensional solution of Eq. (33) under equilibrium conditions, $T=T_{M}$, for which it becomes

$$
\epsilon^{2} \frac{d^{2} \phi}{d x^{2}}-\frac{1}{4 a} g^{\prime}(\phi)=0
$$

with boundary conditions $\phi \rightarrow 0$ as $x \rightarrow-\infty$, and $\phi \rightarrow 1$ as $x \rightarrow+\infty$. The solution is found to be

$$
\phi(x)=\frac{1}{2}\left[\tanh \left(\frac{x}{2 \sqrt{2 a} \epsilon}\right)+1\right]
$$

where we have chosen a constant of integration to locate the interface at $x=0$. From this solution it is clear that the interface has characteristic thickness

$$
\delta=\sqrt{a} \epsilon
$$

To calculate the surface free energy associated with the one-dimensional solution, we employ the Helmholtz free energy functional at temperature $T_{M}$, which from Eq. (4) and Eq. (1) is given by

$$
\mathcal{F}=\mathcal{E}-T_{M} \mathcal{S}=\int_{V}\left[f\left(T_{M}, \phi\right)+\frac{\epsilon^{2} T_{M}}{2}(\nabla \phi)^{2}\right] d v .
$$

The corresponding surface free energy per unit area, which is a surface excess quantity because $f\left(T_{M}, \phi\right)=0$ in the bulk, is

$$
\sigma=\int_{-\infty}^{+\infty} \epsilon^{2} T_{M}\left(\frac{d \phi}{d x}\right)^{2} d x
$$

This may be evaluated to yield

$$
\sigma=\frac{\sqrt{2} \delta T_{M}}{12 a}
$$

Hence, the constant $a$ is related to the material parameters and the interface thickness $\delta$.

In order to proceed further, it is advantageous to nondimensionalize the governing equations. We introduce a length scale $w$ representative of the size of the domain, and use the thermal diffusion time $w^{2} / \kappa$, where $\kappa$ is the assumed constant thermal diffusivity of the liquid, as the reference time scale. Employing these scales, we write

$$
\tilde{\mathbf{x}}=\frac{\mathbf{x}}{w}, \quad \tilde{t}=\frac{t}{w^{2} / \kappa}, \text { and } u=\frac{T-T_{M}}{T_{M}-T_{0}},
$$


where $T_{0}$ is an appropriate reference temperature. Here, a tilde is used to denote the dimensionless equivalent of a dimensional quantity. The governing equations become

$$
\begin{gathered}
{\left[1+[p(\phi)-1] \frac{\tilde{L}^{\prime}(u)}{S}\right] \frac{\partial u}{\partial \tilde{t}}+\frac{\tilde{L}(u)}{S} p^{\prime}(\phi) \frac{\partial \phi}{\partial \tilde{t}}=\tilde{\nabla}^{2} u} \\
\frac{\tilde{\epsilon}^{2}}{m} \frac{\partial \phi}{\partial \tilde{t}}=\tilde{\epsilon}^{2} \tilde{\nabla}^{2} \phi+\tilde{\epsilon} \alpha S \tilde{Q}(u) p^{\prime}(\phi)-\frac{g^{\prime}(\phi)}{4}
\end{gathered}
$$

where

$$
\begin{gathered}
\tilde{L}(u)=L / L_{0}, \\
\tilde{Q}(u)=\int_{0}^{u} \frac{\tilde{L}(\zeta)}{\left[1+\left(\frac{T_{M}-T_{0}}{T_{M}}\right) \zeta\right]^{2}} d \zeta
\end{gathered}
$$

and

$$
\alpha=\frac{\sqrt{2} w\left[L_{0}\right]^{2}}{12 c \sigma T_{M}}, \quad S=\frac{c\left(T_{M}-T_{0}\right)}{L_{0}}, \quad \tilde{\epsilon}=\frac{\delta}{w}, \quad m=\frac{6 \sqrt{2} \delta \sigma}{\tau \kappa T_{M}} .
$$

Below, we determine $m$ explicitly in terms of the interface kinetic coefficient and known material parameters. We shall proceed to discuss Eqns. (40-44) for two different choices of $p(\phi)$ given below.

\section{Phase-Field Models I and II}

In order to proceed in the development of the phase-field model, the function $p(\phi)$ which appears in the energy density must be specified. We consider two different polynomial forms for the function $p(\phi)$ which result in different phase-field models.

Model I: We choose

$$
p(\phi)=\frac{\int_{0}^{\phi} g(\zeta) d \zeta}{\int_{0}^{1} g(\zeta) d \zeta}=\phi^{3}\left(10-15 \phi+6 \phi^{2}\right)
$$

which satisfies the required normalization that $p(0)=0$ and $p(1)=1$ as well as the conditions Eq. (27) and Eq. (28), independent of $Q(T)$, because $p^{\prime}(\phi)=p^{\prime \prime}(\phi)=0$ at $\phi=0$ and 1 . This choice will lead to a phase-field model that we will compare with the one proposed by Langer [2].

Model II: We choose

$$
p(\phi)=\phi^{2}(3-2 \phi)
$$


which satisfies the conditions that $p(0)=0$ and $p(1)=1$, and $p^{\prime}(0)=p^{\prime}(1)=0$; however, because $G^{\prime \prime}(0)-p^{\prime \prime}(0) Q(T)=1 /(2 a)-6 Q(T)$ and $G^{\prime \prime}(1)-p^{\prime \prime}(1) Q(T)=1 /(2 a)+6 Q(T)$, we must require the additional condition that $|12 a Q(T)|<1$ in order to satisfy Eq. (28). This choice, as shown below, leads to a model similar to that proposed by Kobayashi [20].

\subsection{Model I}

Eq. (45) applies and there are no restrictions on $Q(T)$. Therefore, for simplicity, we choose the specific heat of the solid to equal the constant specific heat, $c$, of the liquid, in which case $L(T)=L_{0}$, which results in $\tilde{L}(u) \equiv 1$. We also assume that $\left|T_{M}-T_{0}\right|<<T_{M}$ and so we make the approximation $\tilde{Q}(u)=u$. Thus, Eq. (40) and Eq. (41) become

$$
\begin{gathered}
\frac{\partial u}{\partial \tilde{t}}+\frac{30 g(\phi)}{S} \frac{\partial \phi}{\partial \tilde{t}}=\tilde{\nabla}^{2} u \\
\frac{\tilde{\epsilon}^{2}}{m} \frac{\partial \phi}{\partial \tilde{t}}=\tilde{\epsilon}^{2} \tilde{\nabla}^{2} \phi+30 g(\phi) \tilde{\epsilon} \alpha S u-\frac{1}{4} g^{\prime}(\phi) .
\end{gathered}
$$

The dimensionless parameters $S$ and $\alpha$ can be specified in terms of $w,\left(T_{M}-T_{0}\right)$, and the material parameters. However, the parameters $\tilde{\epsilon}$ and $m$ are still undetermined because they are related to the unknown quantities $\delta$ and $\tau$. We expect the parameter $\delta$ to be much less than $w$. Hence, we fix the parameter $m$ by conducting an asymptotic analysis of the phase-field equations in the limit $\tilde{\epsilon} \rightarrow 0$ with $S, \alpha$, and $m$ of order one. In this limit, a free boundary problem is recovered. The details are analogous to those given by Caginalp [11] and so for brevity we just give the results. The free boundary problem satisfied by the leading order temperature is

$$
\frac{\partial u}{\partial \tilde{t}}=\nabla^{2} u
$$

with interfacial boundary conditions

$$
u=-\tilde{\Gamma}\left(\tilde{\mathcal{K}}+\frac{\tilde{v}_{I}}{m}\right)
$$

and

$$
\left[\frac{\partial u}{\partial n}\right]_{\text {Solid }}^{\text {Liquid }}=-\frac{\tilde{v}_{I}}{S}
$$


where $\tilde{\Gamma}=\sigma T_{m} /\left[w L_{0}\left(T_{M}-T_{0}\right)\right]$ is the dimensionless capillary length, $\tilde{v}_{I}$ is the dimensionless normal velocity of the interface into the liquid and $\tilde{\mathcal{K}}$ is the dimensionless interfacial curvature (measured positive for convex projections into the liquid). The interfacial boundary condition Eq. (50) reduces to the Gibbs-Thomson equation when $\tilde{v}_{I}=0$. The additional term incorporates the effect of interface kinetics. The dimensionless interface kinetic coefficient is therefore represented by $m$; it may be related to the corresponding dimensional quantity $\mu$ by comparison of Eq. (50) with its usual dimensional form:

$$
T=T_{M}-\frac{\sigma T_{M}}{L_{0}} \mathcal{K}-\frac{1}{\mu} v_{I}
$$

which gives

$$
m=\frac{\mu \sigma T_{M}}{\kappa L_{0}} .
$$

In view of Eq. (44), this means that $\tau$ is proportional to $\delta$, which makes $m$ independent of $\tilde{\epsilon}$ as implied by Eq. (41). The only remaining undetermined parameter is therefore $\tilde{\epsilon}$. In practice, $\tilde{\epsilon}$ should be chosen small enough to validate the asymptotics but large enough to make computations practical. This completes the determination of the dimensionless phase-field parameters in terms of the physical parameters.

The advantages of the phase-field model given by Eq. (47) and Eq. (48) are two-fold. First, the model is consistent with the ideas of irreversible thermodynamics because it was derived from an entropy functional, which is the appropriate thermodynamic potential in this nonisothermal situation. Second, it is constructed so that the states $\phi \equiv 0$ and $\phi \equiv 1$ correspond to the bulk solid and liquid phases, respectively, independent of the temperature. This has the advantage that latent heat of the correct total amount is liberated only in the interfacial region. The model proposed by Langer $[1,2]$ can be obtained by replacing the term $30 g(\phi)$, which appears in both Eq. (47) and Eq. (48), by unity, which is its average value over the range $0<\phi<1$, while leaving the term $g^{\prime}(\phi)$ in Eq. (48) as calculated from Eq. (30). This "linearization" would violate Eq. (27) and Eq. (28). Thus, in the Langer model, the bulk solid and liquid phases do not correspond to constant values of $\phi$ independent of the temperature. In a recent model developed by Caginalp and Chen [13], a function $f(\phi)$ was introduced in the free energy functional (their equation 1.6) 
to identify the bulk phases precisely by $\phi= \pm 1$, which is equivalent to maintaining the term $30 g(\phi)$ in Eq. (48), but their model does not have the thermodynamically-consistent $30 g(\phi)$ term in Eq. (47).

\subsection{Model II}

Eq. (46) applies, but there is the restriction $|12 a Q(T)|<1$. Substituting Eq. (46) for $p(\phi)$ and Eq. (30) for $g(\phi)$ into Eq. (40) and Eq. (41) yields the following set of phase-field equations:

$$
\begin{gathered}
\frac{\partial u}{\partial \tilde{t}}-\frac{1}{S}\left[1-\phi^{2}(3-2 \phi)\right] \frac{\partial \tilde{L}(u)}{\partial \tilde{t}}+\frac{6 \tilde{L}(u)}{S} \phi(1-\phi) \frac{\partial \phi}{\partial \tilde{t}}=\tilde{\nabla}^{2} u \\
\frac{\tilde{\epsilon}^{2}}{m} \frac{\partial \phi}{\partial \tilde{t}}=\tilde{\epsilon}^{2} \tilde{\nabla}^{2} \phi+\phi(1-\phi)\left(\phi-\frac{1}{2}+6 \tilde{\epsilon} \alpha S \tilde{Q}(u)\right) .
\end{gathered}
$$

Recently, Kobayashi [20-23] proposed a phase-field model based on the following pair of dimensionless equations:

$$
\begin{gathered}
\frac{\partial u}{\partial \tilde{t}}+\frac{1}{S} \frac{\partial \phi}{\partial \tilde{t}}=\tilde{\nabla}^{2} u \\
\frac{\tilde{\epsilon}^{2}}{m} \frac{\partial \phi}{\partial \tilde{t}}=\tilde{\epsilon}^{2} \tilde{\nabla}^{2} \phi+\phi(1-\phi)\left(\phi-\frac{1}{2}+\frac{\beta}{\pi} \arctan (\nu u)\right) .
\end{gathered}
$$

where $\beta$ and $\nu$ are positive constants with $\beta<1$. The phase-field equation in Model II, Eq. (55), is identical to that suggested by Kobayashi provided that

$$
\tilde{Q}(u)=\frac{\beta}{6 \pi \tilde{\epsilon} \alpha S} \arctan (\nu u) .
$$

Note that our restriction $|12 a Q(T)|<1$ is equivalent to Kobayashi's condition $\beta<1$. The derivative of the function $\tilde{Q}(u)$ is related to the function $\tilde{L}(u)$ by Eq. (43), which gives

$$
\tilde{L}(u)=\frac{\left[1+\left(\frac{T_{M-}-T_{O}}{T_{M}}\right) u\right]^{2}}{1+(\nu u)^{2}},
$$

and $\beta=6 \pi \tilde{\epsilon} \alpha S / \nu$. The function $\tilde{L}(u)$ will have a strong dependence on $u$ unless $\nu$ is chosen to be very small. This dependence on $u$ requires the specific heat, $c_{S}$, of the solid to have a definite dependence on temperature, which, from the derivative of the expression $e_{S}(T)=e_{L}(T)-L(T)$, Eq. (31), and Eq. (42), is represented by

$$
c_{S}=c\left[1-\frac{1}{S} \tilde{L}^{\prime}(u)\right] \text {. }
$$


The quantity $-\tilde{L}^{\prime}(u)$ is plotted in Figure 2 for the case $\nu=10$ (used by Kobayashi) and $\left(T_{M}-T_{0}\right) / T_{M}=0.2$. We note that the corresponding specific heat will vary considerably, even near the melting point, $u=0$, and can possibly become negative. For solidification from a supercooled melt, the temperature in the solid will typically be close to the melting point, with temperature gradients much less than those in the liquid. Nevertheless, there might be problems in using Kobayashi's model for such large values of $\nu$.

Thus, in order to recover the same governing equation for the phase field as Kobayashi, within a thermodynamically-consistent framework, it is necessary for the specific heat of the solid to have the functional dependence on temperature given by Eq. (60). However, the self-consistent equation for the temperature will then be Eq. (54) instead of Kobayashi's Eq. (56). We first compare the terms containing $\partial \phi / \partial \tilde{t}$; they will only be important in the interfacial region where $u \sim 0$ so $\tilde{L}(u) \sim 1$. Thus, replacing $6 \phi(1-\phi)$ by its average value of 1 over the range $0<\phi<1$ reproduces Kobayashi's corresponding term. This is similar to the "linearization" required to recover Langer's model from Model I. The term in Eq. (54) containing $\partial \tilde{L} / \partial \tilde{t}$, however, is completely absent in Kobayashi's Eq. (56). It vanishes in the liquid where $\phi=1$, but not in the solid where $\phi=0$, and arises because of the variable specific heat necessary to make Kobayashi's model thermodynamically consistent. In fact, for $\phi=0$, Eq. (60) can be used to combine the first two terms on the left-hand side of Eq. (54) to yield

$$
\frac{c_{S}}{c} \frac{\partial u}{\partial \tilde{t}}=\tilde{\nabla}^{2} u
$$

which is in complete agreement with the classical equation in the solid phase.

An alternative approach to comparing our model to that of Kobayashi would be to choose $c_{S}$, and $p(\phi)$ to achieve the same equation for the temperature. This would require $c_{S}=c$ and $p(\phi)=\phi$, but then $G^{\prime}(\phi)-p^{\prime}(\phi) Q(T)=G^{\prime}(\phi)-L_{0}\left[\left(1 / T_{M}\right)-(1 / T)\right]$, and Eq. (27) would require $G(0)$ and $G(1)$ to depend on temperature, which is a contradiction. 


\section{Discussion}

We have employed the method of Penrose and Fife, based on an entropy functional, to derive a class of phase-field models for the crystallization of a pure material from its melt. This method provides a Lyapunov functional [25] which may facilitate the development of numerical solution methods. The parameters of the model are selected by passing to the asymptotic limit of a sharp interface and comparing with the classical model. Criteria are developed to ensure that the phase field will take on fixed values, independent of temperature, for bulk solid and liquid. Specific choices of the phase-field function are made to yield models, which we call Model I and Model II, that bear strong resemblances to the models of Langer and Kobayashi, respectively. However, Models I and II contain nonlinear functions of the phase field that are absent in the models of Langer and Kobayashi, but are necessary to guarantee thermodynamic consistency. Model I differs from Langer's model only in the interfacial region. Model II differs from Kobayashi's model both in the interfacial region and in the bulk solid; in particular, the equation for the temperature that is thermodynamically consistent with Kobayashi's equation for the phase field has a specific heat in the solid phase that is a rather strong function of temperature, even for temperatures close to the melting point.

These models also guarantee positive entropy production locally in space. The volumetric entropy production due to heat flow, $|\mathrm{q}|^{2} / M_{T}$, and the entropy flux, $\mathrm{q} / T$, are classical and require no further discussion. The corresponding quantities, $\tau(\dot{\phi})^{2}$ and $\epsilon^{2} \dot{\phi} \nabla \phi$, require further clarification, which we proceed to give by relating them to analogous quantities for a sharp-interface model. The volumetric entropy production $\tau(\dot{\phi})^{2}$ will contribute only in volume elements $d v$ in which $\dot{\phi}$ differs appreciably from zero. These elements will correspond to regions through which a solid-liquid interface is passing, and for which $\dot{\phi} \sim v_{I} / \eta$ and $d v \sim \eta d a_{I}$, where $d a_{I}$ is an element of area on the interface. Here we have taken $\eta=6 \sqrt{2} \delta$ as a more precise representation of the interface thickness; from Eq. (35), $\eta$ 
represents the distance over which $\phi$ varies from roughly 0.05 to 0.95 . Thus,

$$
\tau(\dot{\phi})^{2} d v \sim \frac{\tau}{\eta} v_{I}^{2} d a_{I}=\frac{L_{0}}{\mu T_{M}^{2}} v_{I}^{2} d a_{I}
$$

where Eqs. (44) and (53) have been used. The quantity $\left[L_{0} /\left(\mu T_{M}^{2}\right)\right] v_{I}^{2}$ turns out to be the entropy production per unit area associated with the movement of a sharp interface for a linear kinetic law of the form represented by Eq. (52), as can be shown using the methodology of Gurtin [26].

Similarly, the entropy flux, $\epsilon^{2} \dot{\phi} \nabla \phi$, will only contribute to Eq. (6) over area elements $d a$ of the control volume through which an interface passes and has a component of its normal growth velocity $\mathbf{v}_{\mathbf{I}}$ along $\mathbf{n}$. Identifying $\dot{\phi}$ as above and noting that $\nabla \phi \sim-\mathbf{v}_{\mathbf{I}} /\left(v_{I} \eta\right)$, we see that

$$
\epsilon^{2} \dot{\phi} \nabla \phi \cdot \mathbf{n} \sim-\frac{\epsilon^{2}}{\eta^{2}} \mathbf{v}_{\mathbf{I}} \cdot \mathbf{n}=-\frac{\sigma}{\eta T_{M}} \mathbf{v}_{\mathbf{I}} \cdot \mathbf{n},
$$

where Eq. (36) and (39) have been used to eliminate $\epsilon$. For an interface corresponding to Eq. (35), one can show from Eq. (1) that there is no surface excess internal energy per unit area relative to a sharp interface located at $x=0$. Therefore, $\sigma=-T_{M} s^{x s}$, where $s^{x s}$ is the surface excess entropy per unit area relative to a sharp interface located at $x=0$. Thus we have

$$
\epsilon^{2} \dot{\phi} \nabla \phi \cdot \mathbf{n} d a \sim \frac{s^{x s}}{\eta} \mathbf{v}_{\mathbf{I}} \cdot \mathbf{n} d a .
$$

The area over which this term will contribute to Eq. (6) is a thin ribbon defined by the intersection of the diffuse interface with the area $\mathcal{A}$ of the control volume. Hence the area element $d a \sim d \ell \eta \csc \Theta$, where $d \ell$ is an element of length along the ribbon and $\Theta$ is the angle between $\mathbf{v}_{\mathbf{I}}$ and $\mathbf{n}$. This trigonometric factor arises because the diffuse boundary is not necessarily perpendicular to the surface of the control volume. Thus finally

$$
\int \epsilon^{2} \dot{\phi} \nabla \phi \cdot \mathbf{n} d a \sim \oint s^{x s} v_{I} \cot \Theta d \ell
$$

which is in complete agreement with the analysis of Gurtin for a sharp interface model $[27,28]$. Eq. (65) represents the rate at which entropy exits the control volume by means of the passage of portions of the interface out of the surface of the control volume. It makes 
no contribution in regions of that surface at which the interface is moving in a direction perpendicular to the normal of the control volume.

Penrose and Fife were able to obtain Langer's model from a formalism that guarantees positive entropy production by making the following choices (in our notation, equivalent to their Eqn. 3.11):

$$
p(\phi)=\phi, \quad L(T)=L_{0}, \quad e_{S}(T)=A-B / T,
$$

where $A$ and $B$ are constants. This choice of $p(\phi)$, in view of Eq. (27), would require $G(\phi)$ to depend on $T$, a contradiction. Therefore, for their model, the values of $\phi$ corresponding to the bulk phases will depend on temperature.

The methodology developed in the present paper can be extended to develop a phasefield model for the non-isothermal solidification of an alloy. For a binary alloy, for example, it would be necessary to specify the internal energy as a function of $T, \phi$, and the composition $c$. This would be a logical extension of the phase-field model for isothermal binary alloy solidification, developed for a lens-type phase diagram in Ref. [29]. Another extension, which will be the subject of a forthcoming paper, is to incorporate arbitrary anisotropy of surface tension and interface kinetics in a thermodynamically-consistent manner.

\section{Acknowledgements}

The authors are grateful for discussions with W. J. Boettinger. This work was performed with support from a NATO collaborative grant, the Applied and Computational Mathematics Program of DARPA, the Microgravity Science and Applications Program of NASA, and the National Science Foundation, under grant DMR-9211276. One of the authors (R.J.B.) was supported by a National Research Council Postdoctoral Fellowship. 


\section{Figure Captions}

Figure 1. Sketch of the internal energies $e_{L}(T)$ and $e_{S}(T)$ as functions of temperature. The solid curves correspond to the stable bulk phases, whereas the dashed curves correspond to either supercooled liquid or superheated solid. The difference between the curves at any temperature is the function $L(T)$, which is equal to the latent heat $L_{0} \equiv L\left(T_{M}\right)$ at the melting point $T_{M}$.

Figure 2. Plot of the quantity $-\tilde{L}^{\prime}(u)$ versus $u$ that illustrates (see Eq. (60)) the temperaturedependence of the specific heat of the solid, $c_{S}$, that is necessary to obtain a thermodynamicallyconsistent equation for temperature corresponding to Kobayashi's model. The plot is for $\nu=10$, used by Kobayashi in his computations, and $\left(T_{M}-T_{0}\right) / T_{M}=0.2$. For values of the undercooling with $S<1$, there will be a rather large variation of $c_{S}$ with temperature, even for temperatures near the melting point. 


\section{References}

[1] J. S. Langer, private communication, August, 1978.

[2] J. S. Langer, in Directions in Condensed Matter Physics, edited by G. Grinstein and G. Mazenko, (World Scientific, Philadelphia, 1986) p. 165.

[3] B. I. Halpern, P. C. Hohenberg and S-K. Ma, Phys. Rev. B10, (1974) 139.

[4] J. B. Collins and H. Levine, Phys Rev. B31 (1985) 6119.

[5] G. Caginalp, in Applications of Field Theory to Statistical Mechanics, edited by L. Garrido, Lecture Notes in Physics No. 216 (Springer-Verlag, Berlin, 1985) p. 216.

[6] G. Caginalp, in Material Instabilities in Continuum Problems and Related Mathematical Problems, edited by J. M. Ball (Oxford University Press, Oxford, 1988) p 35.

[7] G. Caginalp and P. C. Fife, Phys. Rev. B33 (1986) 7792.

[8] G. Caginalp, Arch. Rat. Mech. Anal. 92 (1986) 205.

[9] G. Caginalp, Annals of Physics 172 (1986) 136.

[10] G. Caginalp and P. C. Fife, SIAM J. Appl. Math 48 (1988) 506.

[11] G. Caginalp, Phys. Rev. A 39 (1989) 5887.

[12] G. Caginalp and E. A. Socolovsky, J. Comp. Phys. (1991) 85.

[13] G. Caginalp and X. Chen, in On the Evolution of Phase Boundaries, edited by M. E. Gurtin and G. B. McFadden, The IMA Volumes in Mathematics and Its Applications Vol. 43 (Springer-Verlag, New York, 1992) p. 1.

[14] M. E. Gurtin, in Metastability and Incompletely Posed Problems, edited by S. Antman, J. L. Ericksen, D. Kinderlehrer and I. Muller, The IMA Volumes in Mathematics and Its Applications Vol. 3, (Springer-Verlag, New York, 1987) p. 135.

[15] P. C. Fife and G. S. Gill, Physica D35 (1989) 267. 
[16] P. C. Fife and G. S. Gill, Phys. Rev, A43 (1991) 843.

[17] G. J. Fix, in Free boundary problems: theory and applications Vol. II, edited by A. Fasano and M. Primicerio (Pitman, Boston, 1983) 580.

[18] J-T. Lin, The Numerical Analysis of a Phase Field Model in Moving Boundary Problems, Doctoral Thesis, Department of Mathematics, Carnegie Mellon University (1984).

[19] S. A. Schofield and D. W. Oxtoby, J. Chem. Phys. 94 (1991) 2176.

[20] R. Kobayashi, Bull. Jpn. Soc. Ind. Appl. Math. 1 (1991) 22.

[21] R. Kobayashi, Computing Optimal Geometries, Proceedings of an AMS Special Session, edited by Jean Taylor, videotapes (1991).

[22] R. Kobayashi, Modeling and Numerical Simulations of Dendritic Crystal Growth, to appear in Physica D (1992).

[23] R. Kobayashi, Simulations of Three Dimensional Dendrites, preprint and associated video tape.

[24] A. A. Wheeler, B. T. Murray, and R. J. Schaefer, Computation of Dendrites Using a Phase-Field Model, to appear in Physica D (1992).

[25] O. Penrose and P. C. Fife, Physica D43 (1990) 44.

[26] M. E. Gurtin, Arch. Rational Mech. Anal. 104 (1988) 195.

[27] M. E. Gurtin, Arch. Rational Mech. Anal. 96 (1986) 199.

[28] M.'E. Gurtin, in PDE's and Continuum Models of Phase Transitions, edited by M. Rascle, D. Serre, and M. Slemrod, Lecture Notes in Physics No. 344 (Springer-Verlag, New York, 1989) p. 99.

[29] A. A. Wheeler, W. J. Boettinger and G. B. McFadden, Phys. Rev. A45 (1992) 7424. 


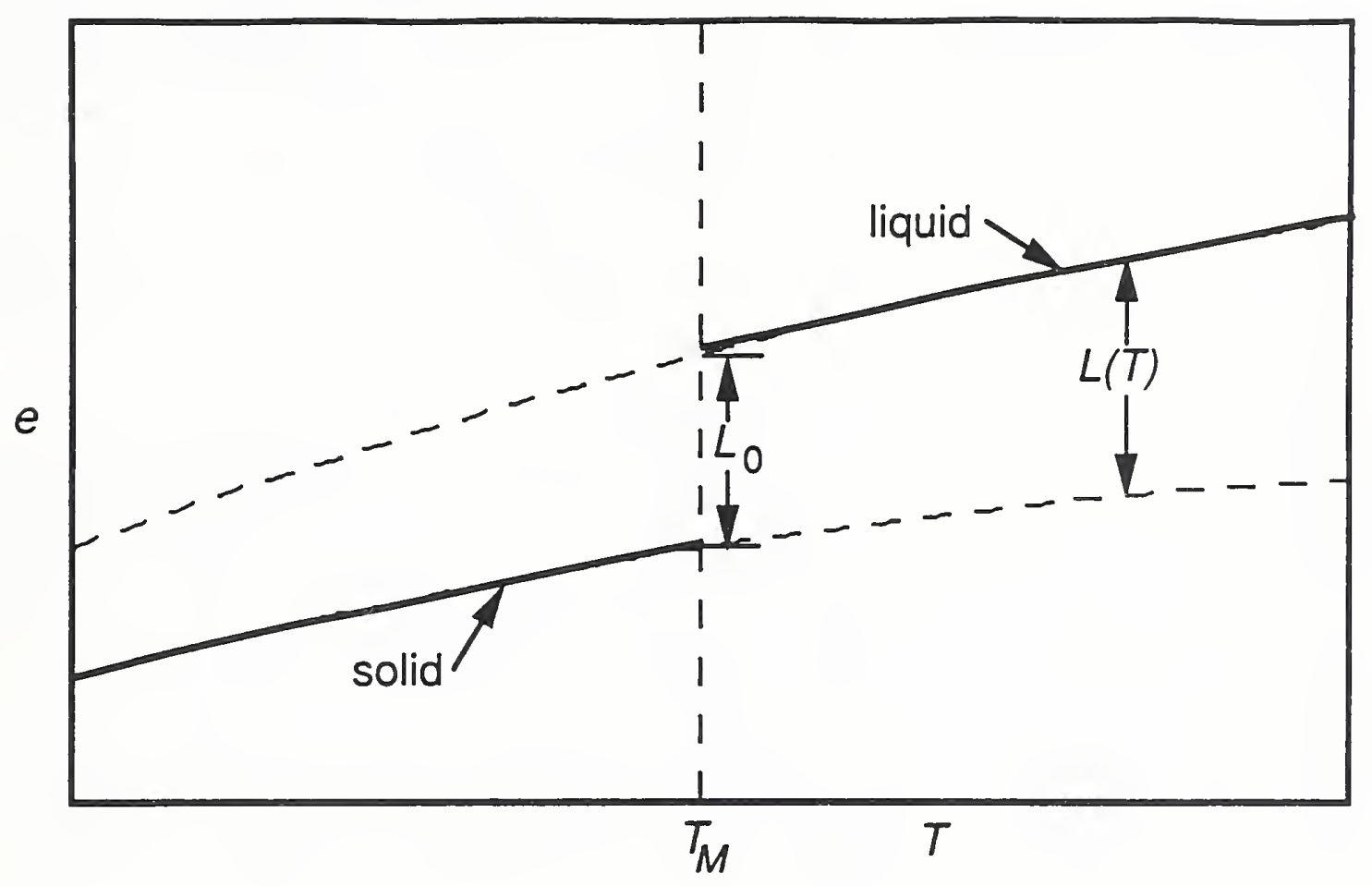

Figure 1 . 


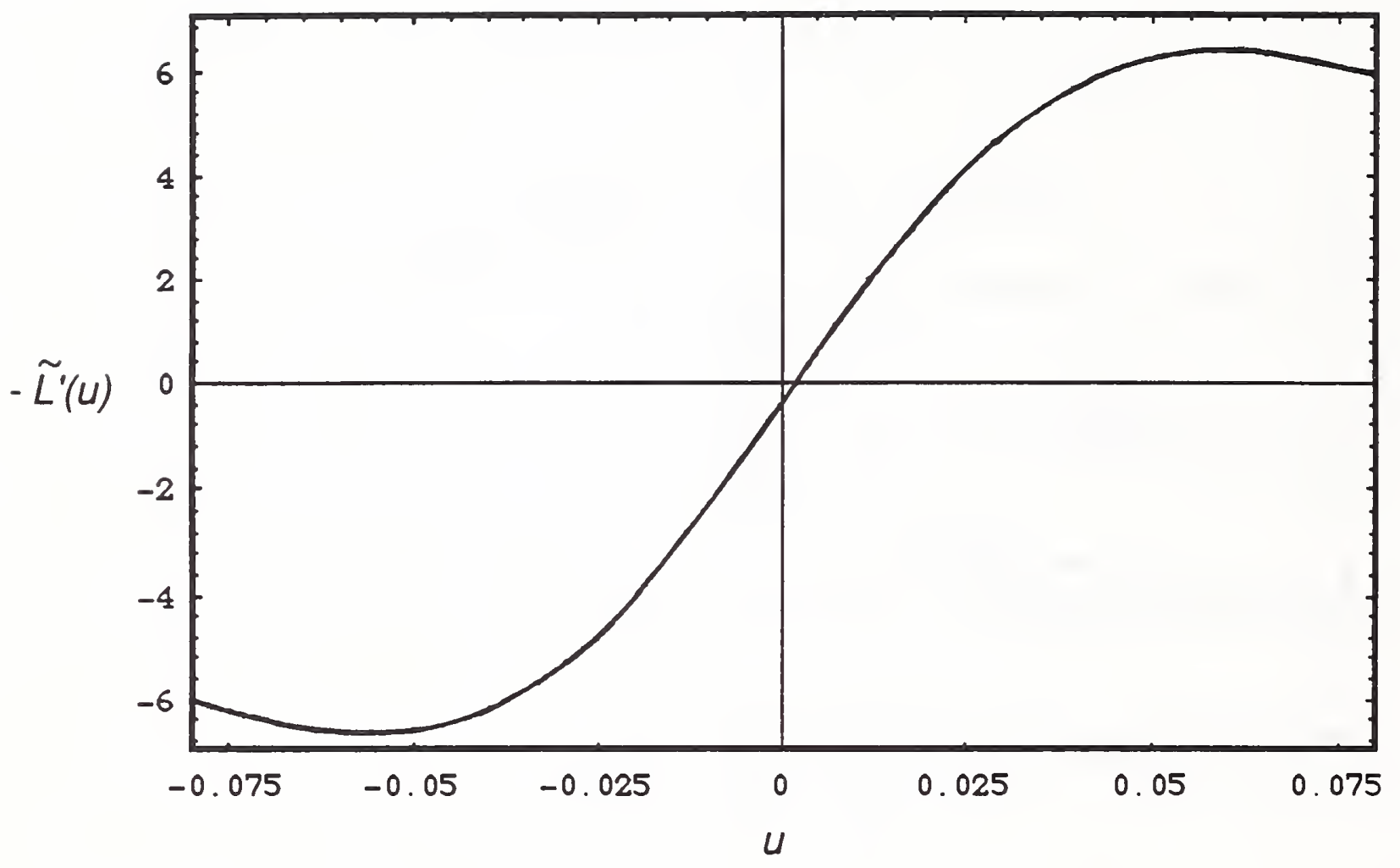

Figure 2. 


\begin{tabular}{|c|c|}
\hline \multirow{4}{*}{$\begin{array}{l}\text { U.S. DEPARTMENT OF COMMERCE } \\
\text { NATIONAL INSTITUTE OF STANDARDS AND TECHNOLOGY }\end{array}$} & 1. PUBUCATION OA REPOAT NUMBEA \\
\hline & $\begin{array}{l}\text { N I S T I R } 4956 \\
2 \text { PERFORMIMO ORGANIZATION AEPORT MUMBER }\end{array}$ \\
\hline & \\
\hline & $\begin{array}{l}\text { 3. PUBLCATION DATE } \\
\text { NOVEMBER } 1992\end{array}$ \\
\hline \multicolumn{2}{|l|}{ 4. TMLLE AND SUBTITLE } \\
\hline \multicolumn{2}{|l|}{ Thermodynamically-Consistent Phase-Field Models for Soldification } \\
\hline \multicolumn{2}{|c|}{$\begin{array}{l}\text { 5. AUTHOR(S) } \\
\text { S-L Wang, R.F. Sekerka, A.A. Wheeler, B.T. Murray, S.R. Coriell, R.J. Braun, and } \\
\text { G.B. McFadden }\end{array}$} \\
\hline \multirow{3}{*}{$\begin{array}{l}\text { 5. PERFOAMINO OAGANIZATION (IF JOINT OA OTHEA THAN NIST, SEE INSTRUCTIONS) } \\
\text { U.S. DEPARTMENT OF COMMERCE } \\
\text { MATIONAL INSTITUTE OF STANDARDS AND TECHMOLOQY } \\
\text { GANTHEASBURO, MD } 20899\end{array}$} & 7. CONTRACT/OAAMT NUMBER \\
\hline & \\
\hline & 8. TYPE OF REPORT AND PERIOD COVERED \\
\hline \multicolumn{2}{|l|}{ 9. SPONSORING ORQANIZATION MAME AND COMPLETE ADDRESS (STAEET, CITY, STATE, ZIP) } \\
\hline \multicolumn{2}{|l|}{$\begin{array}{l}\text { National Institute of Standards and Technology } \\
\text { Bldg. 101, Room A238, Cloppers Road } \\
\text { Gaithersburg, MD } 20899\end{array}$} \\
\hline
\end{tabular}

\section{SUPPLEMENTARY NOTES}

11. ABSTRACT (A 200-WORD OR LESS FACTUAL SUMMAAY OF MOST SIONIFICANT INFORMATION. BF DOCUMENT INCLUDES A SIQNIFICANT BIBLOORAPHY OR
LTERATURE SUAVEY, MENTION TITERE) In an effort to unify the various phase-field models that have been used to study solidification, we have developed a class of phase-field models for crystallization of a pure substance from its melt. These models are based on an entropy functional, as in the treatment of Penrose and Fife, and are therefore thermodynamically consistent inasmuch as they guarantee spatially local positive entropy production. General conditions are developed to ensure that the phase field takes on definite values, independent of temperature, for the bulk phases. resemblances to the models proposed by Langer and Kobayashion tho models that bear strong nonlinear functions of the phase field that are and Kobayashi. Our models contain additional consistency.

KEY WORDS (O TO 12 ENTRIES; NPPLABETICAL ORDER; CAPTA AIE ONLY PAOPER MMMES; AND SEPARATE KEY WOADS DY SEMICOLONS) crystal growth, entropy production, non-equilibrium thermodynamics, phase field, solidification, solid-liquid interface

FOA OFFCLAL DISTRIBUTION. DO NOT REEASE TO MATIOMAL TECHMICAL MFORLATION SERVCE (NTIS). OROER FROM SUPERINTENDENT OF DOCUMENTS, U.S. COVEANMENT PAINTINO OFFICE,
WASHINOTON, DC 20402 . 

